\title{
CONCILIACIÓN DE LA VIDA FAMILIAR Y LABORAL ${ }^{1}$
}

\author{
SOL RUIZ DE LA CUESTA FERNÁNDEZ \\ IRENE BAJO GARCÍA \\ Universidad de Alicante
}

\section{PRESENTACIÓN}

A la hora de abordar una materia como ésta, en el contexto de un Curso organizado por y para juristas, era dudoso qué enfoque resultaría el más adecuado, pues, como se ha dicho, la naturaleza principalmente jurídica de este encuentro invitaba a optar por un tratamiento puramente jurídico de la conciliación entre la vida familiar y laboral. Sin embargo, una perspectiva jurídica pura del tema resulta excesivamente descriptiva y mucho menos apasionada que la que se desprende de la combinación de factores jurídicos y sociales. Por ello, hemos decidido abordar la materia desde la dimensión sociolaboral del trabajo de la mujer, considerando "trabajo» tan sólo a aquel que se realiza por cuenta ajena, es decir, según los parámetros previstos por el Estatuto de los Trabajadores $^{2}$ (ET, en adelante), sin atender, por lo tanto, a las particularidades derivadas de una prestación de servicios en régimen autónomo.

1. El presente estudio, articulado y revisado conjuntamente, corresponde por lo que se refiere a su elaboración escrita a la Profesora Sol Ruiz de la Cuesta los epígrafes 1, 3 y 4, y a la Profesora Irene Bajo los epígrafes 2 y 5. Queremos también dejar constancia de que la conciliación entre la vida familiar y laboral está pendiente de sufrir un -se espera que importante-cambio a nivel legislativo, pues en estos momentos existen dos proyectos de ley de obligada referencia. Por un lado, el de Promoción de la Autonomía Personal y Atención a las Personas en situación de Dependencia, que superó su primer trámite en el Congreso el pasado 23 de junio de 2006, con los votos favorables de PSOE, ERC e IU-ICV. Por otro lado, el Consejo de Ministros aprobó el 26 de junio de 2006 la remisión a las Cortes Generales del Proyecto de Ley Orgánica de Igualdad entre Hombres y Mujeres.

2. Real Decreto Legislativo 1/1995, de 24 de marzo, por el que se aprueba el Texto Refundido de la Ley del Estatuto de los Trabajadores (BOE de 29 de marzo). El artículo $1.1 \mathrm{ET}$, al delimitar el ámbito de aplicación de la Ley, establece los presupuestos sustantivos del contrato de trabajo, a saber: voluntariedad, dependencia, ajenidad y retribución, cuando indica que "La presente Ley será de aplicación a los trabajadores que voluntariamente presten sus servicios retribuidos por cuenta ajena y dentro del ámbito de organización y dirección de otra persona, física o jurídica, denominada empleador o empresario». 
Con la exposición que ahora iniciamos se persiguen diversos objetivos. En primer lugar, el de dejar constancia, siquiera brevemente, de cuál ha sido el proceso de incorporación de la mujer al mundo del trabajo, en función de qué factores y bajo qué concretas condiciones. A continuación, se analizarán las medidas con las que el ordenamiento jurídico laboral pretende garantizar la conciliación entre familia y trabajo. Seguidamente, se valorará la eficacia real de estas medidas, intentando identificar las razones por las que, en su caso, puedan resultar insuficientes o inoperantes a la hora de conseguir el objetivo que persiguen. Finalmente, se intentarán identificar los retos y los cambios a los que debe hacerse frente si se quiere hablar en el futuro de una plena conciliación entre vida familiar y vida laboral.

\section{LA PROGRESIVA INCORPORACIÓN DE LA MUJER AL TRABAJO}

Comenzando por el proceso de incorporación de la mujer al mundo del trabajo éste se inicia en nuestro país recientemente, aunque debe señalarse que con una progresión notable. En un estadio previo la sociedad española se muestra como una sociedad eminentemente patriarcal, caracterizada por una división sexual del trabajo y por contemplar roles claramente diferenciados para hombre y mujer. En esta sociedad las mujeres quedan relegadas al ámbito de lo doméstico y privado, en tanto el hombre ocupa el rol de proveedor, inserto en el ámbito de lo público. Es evidente que, en este contexto, resulta innecesario totalmente plantear siquiera una política de conciliación de la vida familiar y laboral.

Este modelo ha funcionado durante un largo período de tiempo, y no es hasta prácticamente las dos últimas décadas cuando la mujer comienza a introducirse de una manera paulatina pero constante e irreversible en el mercado de trabajo. Los factores que han contribuido a dicha incorporación son múltiples. Así, la prosperidad económica que ha caracterizado la evolución de nuestro país en las últimas décadas multiplica la necesidad de mano de obra de las empresas $y$, al mismo tiempo, permite a las familias una ampliación del número de sus miembros que acceden a una formación académica. Junto a ello ha de situarse la aprobación de la Ley del divorcio, que amplía la capacidad de decisión de la mujer y genera para ésta una necesidad de estabilidad económica que el matrimonio ya no garantiza ${ }^{3}$. Por otra parte, los ingresos en la familia que proporciona el trabajo de la mujer permiten elevar el nivel de vida y consumo de la misma, situación a la que ya no se renuncia. Así mismo, la propia llegada de la democracia propicia una lenta pero pacífica aceptación social de la autonomía económica y sentimental de la mujer. En ello ha influido definitivamente la propia actitud de la mujer, que ha mostrado su autonomía personal y su inquietud laboral a través de su incorporación al mercado de trabajo con independencia

3. La mayor igualdad real entre hombre y mujer genera nuevos modelos conyugales en los que el amor y no la necesidad determinan tanto la celebración como la conservación del matrimonio. 
del grado de necesidad de los ingresos con ello obtenidos para su sustento o el de su familia.

Propiciada por los factores descritos, la incorporación de la mujer al trabajo se produce de manera no traumática ni conflictiva, hecho que probablemente determine que la misma no haya venido acompañada de un paralelo cambio en el modelo social heredado y anteriormente descrito. En este sentido, tanto desde las iniciativas privadas como desde las públicas se ha enfatizado en la incorporación de la mujer a la vida laboral, quedando absolutamente postergada la imprescindible y paralela democratización de la vida doméstica, esto es, que los varones asuman la parte de responsabilidad que les corresponde en el reparto igualitario de tareas domésticas. La corresponsabilidad familiar y el nuevo papel que el hombre ha de desempeñar en el seno de la misma no ha sido asumida ni por los varones ni por la sociedad en general ${ }^{4}$. La inadaptación al nuevo modelo de relaciones familiares, en casos extremos, da lugar a graves conflictos en el seno de las familias, como es el caso de la violencia doméstica, los menores conflictivos, etc., cuyo estudio corresponde a la sociología.

A ello ha de sumarse la incidencia de políticas neoliberales, con la consecuente reducción del estado de bienestar y cuyo resultado es el llamado modelo familista, propio de los países mediterráneos (España, Grecia e Italia), en el que el Estado da por supuesto y pone énfasis en que los hogares asuman la obligación de garantizar el bienestar de sus miembros, que no depende del estado ni del trabajo y que se estructura en torno a la tradicional división de roles.

La suma de estos elementos ofrece como resultado una incorporación efectiva de la mujer al mundo laboral que no ha venido acompañada de una reducción de sus tareas familiares, consistente fundamentalmente en el cuidado de los hijos y otros familiares, junto a la gobernación del hogar familiar, asumiendo todas aquellas funciones familiares que no ejecuta el hombre y todas aquellas asistenciales que no proporciona el Estado (centros de atención de menores y niños, y de personas mayores, fundamentalmente).

Desde la perspectiva jurídico-laboral, la situación descrita desemboca en tres grandes problemas. En primer lugar, la mujer trabajadora se encuentra sometida a una doble jornada laboral, esto es, la que desarrolla en su trabajo por cuenta ajena, sumada a la que desarrolla en su casa, al cuidado de su familia. Con el desequilibrio añadido de que ese trabajo doméstico, que no es remunerado ni tiene atribuido un valor económico, está, sin embargo, subsidiando el crecimiento, pues es la mujer quien, al hacerse cargo de esas responsabilidades gratuitamente, permite que se genere riqueza que no es necesario invertir en el cuidado y atención de la familia. En segundo lugar, y consecuencia directa de la doble jornada de la mujer trabajadora, se produce una feminización del desempleo. En tercer lugar, se generan y perpetúan situaciones de discriminación en

4. Ciertamente, en la última década se ha percibido un aumento de la participación de los hombres en las tareas domésticas; sin embargo, ello no permite eliminar su carácter marginal y minoritario. 
el trabajo, tanto salarial como ocupacional, reflejada esta última en la dificultad para acceder a puestos de responsabilidad en las empresas.

Frente a la situación descrita, resulta positivo constatar la existencia de una conciencia social respecto a las dificultades del acceso a la vida laboral de la mujer, conciencia que, desde la perspectiva jurídico-laboral se traduce en iniciativas legislativas destinadas a promover condiciones de igualdad. Su análisis es objeto del siguiente apartado.

\section{MARCO LEGISLATIVO}

No existe en nuestro ordenamiento jurídico una legislación integral en torno a la conciliación de la vida familiar y laboral, sino que el contenido que integra ésta se encuentra regulado en diversas normas. Sin duda, la Ley 39/99 de conciliación de la vida laboral y familiar constituye el texto que más directa y exclusivamente afronta la materia. No obstante, junto a esta norma deben citarse otras, como es la Constitución, el Estatuto de los Trabajadores o la Ley de Prevención de Riesgos Laborales ${ }^{6}$.

Dada la estrecha vinculación de la conciliación de vida laboral y familiar con la igualdad de oportunidades y la protección de la familia, son varios los preceptos constitucionales cuyo contenido regulador afecta a aquella materia. Así, el art. 9.2 CE dirige un mandato a los poderes públicos para la promoción de condiciones efectivas de igualdad. La protección social, jurídica y económica de la familia se consagra en el art. $39.1 \mathrm{CE}$, precepto cuyo contenido debe compatibilizarse con el derecho al trabajo, a una promoción en el mismo y a una remuneración suficiente sin discriminación (entre otras) por razones de sexo, contemplado en el art. 35.1 CE. Con una vinculación de carácter indirecto, también cabe citar la obligación de los poderes públicos del mantenimiento de un régimen público de seguridad social, especialmente en lo relativo a la protección por desempleo (art. $41 \mathrm{CE}$ ).

El ET reitera el mandato constitucional de no discriminación en las relaciones laborales, entre otras causas, por razones de sexo. Junto a esta genérica proclamación del derecho a la igualdad, son varias las previsiones que pueden contemplarse en la norma estatutaria dirigidas a la conciliación de la vida laboral y familiar? ${ }^{7}$. El conjunto de previsiones abarca al permiso por lactancia de hijo menos de nueve meses, de una hora de duración (o reducción de la jornada en media hora) (art. 37.4 ET); el permiso por hospitalización de hijo prematuro,

5. Vid., en el mismo sentido, ARGÜElles Blanco, Ana Rosa: «La igualdad de oportunidades mediante la conciliación de la vida laboral y familiar», en AAVV: Igualdad de oportunidades y responsabilidades familiares", Madrid, CES, 2004, p. 21.

6. No es éste el marco apropiado para realizar un análisis exhaustivo del marco legislativo en materia de conciliación. Al respecto, se remite a GIL SUÁREZ, Luis: «La conciliación de la vida familiar y laboral de las personas trabajadoras. Puntos críticos», en AAVV: Nueva sociedad y derecho del trabajo, Madrid, Ministerio de Trabajo y Asuntos Sociales/La Ley, 2004, pp. 553 y ss.

7. Para un análisis minucioso de cada una de las previsiones estatutarias al respecto, consultar la obra colectiva Igualdad de oportunidades y responsabilidades familiares, Madrid, CES, 2004. 
de duración idéntica al anterior pero sujeto a reducción de salario (art. 37.4 bis); la reducción de jornada entre $1 / 3$ y $1 / 2$, con equivalente reducción de salario, por guarda legal de un menor de seis años; el derecho a la movilidad geográfica en base a la reagrupación familiar (art. 40.3 ET) ${ }^{8}$; la suspensión del contrato de trabajo por maternidad, riesgo durante el embarazo o adopción (art. 45.1.d); la excedencia para el cuidado hijos menores de tres años, que conlleva reserva del puesto de trabajo durante el primer año y, los siguientes, un derecho preferente al reingreso (art. $46.3 \mathrm{ET}$ ); la licencia por maternidad de 16 semanas, a distribuir según voluntad de la madre, que puede optar por ceder parte del permiso al padre, a excepción de las seis semanas posteriores al parto (art. 48.4 ET); la suspensión del contrato de trabajo por riesgo durante el embarazo (art. $48.5 \mathrm{ET}$ ), y la declaración de nulidad del despido cuando el mismo se produzca durante y con causa en el embarazo o durante la suspensión del contrato de trabajo por alguna de las causas expuestas y relacionadas con la maternidad?.

Contribuye al acervo normativo en la materia la Ley de Prevención de Riesgos Laborales, cuando en su art. 26 impone al empresario la obligación de llevar a cabo una evaluación específica de los riesgos que puedan afectar a la mujer embarazada o puérpera, y, junto a ello, la obligación de adaptar las condiciones y jornada de trabajo a esta circunstancia, o bien al traslado a la mujer a un puesto de trabajo compatible con su estado.

Por su parte, la Ley 39/99 de conciliación de la vida laboral y familiar, ha cumplido la importante tarea de hacer aflorar la problemática que dicha conciliación plantea e incorporarla al debate social. Sin embargo, su contenido regulador ha sido absorbido por los textos anteriormente enumerados, cuando, probablemente, el legislador debía haber convertido dicha Ley en la norma de referencia en materia de conciliación ${ }^{10}$.

En cualquier caso, resulta fundamental conocer que la totalidad de las medidas previstas en los preceptos legales enumerados tienen atribuido por el ordenamiento jurídico el carácter de mínimos, esto es, regulaciones que pueden ser mejoradas tanto a través de los convenios colectivos como de los contratos

8. Rabanal Carbajo menciona ésta como ejemplo de medida de conciliación escasamente explorada. (RABANAl CARBAjo, Pedro: «Familia y trabajo. Algunas reflexiones sobre el ordenamiento laboral y la conciliación de la vida laboral y familiar», en AAVV: Nueva sociedad y derecho del trabajo, op.cit., p. 57.)

9. Es escasa la aportación jurisprudencial respecto al análisis de los aspectos conflictivos que pueda plantear esta regulación. Sea como fuere, para una relación y análisis de las principales cuestiones polémicas planteadas y resueltas a nivel jurisprudencial, vid. AGUILERA IZOUIERDO, Raquel; CRISTÓbal Roncero, Rosario y GaRcía PiñeIRO, Nuria: "La conciliación de la vida familiar y laboral: cuestiones polémicas en la reciente doctrina jurisprudencial», en AAVV.: Nueva sociedad y derecho del trabajo, op. cit., pp. 583 y ss.

10. Como indica el profesor De La Villa, al constituirse en una ley de reforma de otras anteriores ha renunciado a un planteamiento de conjunto sobre el fenómeno de la conciliación. Sin embargo, no existe en el derecho español una filosofía de la conciliación de la vida familiar y laboral. DE LA VILla GIL, Luis Enrique: «El marco normativo jurídico laboral vigente sobre conciliación trabajo/familia. Presente y futuro", en AAVV: La conciliación entre el trabajo y la familia, op. cit., p. 70 . 
de trabajo. Posibilidad que, como se verá más adelante, no ha sido convenientemente aprovechada por los agentes sociales.

\section{LA EFICACIA DE LAS MEDIDAS DESTINADAS A LA CONCILIACIÓN DE LA VIDA FAMILIAR Y LABORAL}

Acaban de exponerse las diferentes medidas que el ordenamiento jurídico laboral arbitra con el objetivo de conciliar la vida familiar y laboral. Cabe preguntarse, sin embargo, hasta qué punto son realmente eficaces y cuáles son, en su caso, los obstáculos con los se encuentra la aplicación práctica de las mismas.

Los datos con los que se cuenta en este momento ${ }^{11}$ arrojan cifras que, a priori, hacen pensar que la batería de medidas descrita no alcanza el objetivo que persigue. Si, como se desprende de las cifras, las mujeres con hijos menores de 15 años trabajan en menor número que las que no los tienen o las que los tienen de más edad, no es difícil concluir que a la mujer española no le resulta sencillo conciliar familia y trabajo. Por otro lado, se constata que los permisos de maternidad los solicitan y disfrutan, en un $98 ' 46 \%$, las mujeres, frente al escasísimo 1'54\% de hombres que lo hacen, con una evolución del disfrute por parte de los padres de tan sólo el 0'17\% en los últimos cuatro años ${ }^{12}$. Del mismo modo, las excedencias por cuidado de hijos/as las solicitan mayoritariamente las madres (96'38\%, frente a un 3'98\% de padres) y en los últimos cuatro años sólo ha aumentado en un $0^{\prime} 76 \%$ el número de padres que las disfrutan.

Desde luego, la propia configuración de las medidas explica por sí misma alguno de los datos transcritos. El hecho de que su disfrute, las más de las veces, implique reducción o pérdida de salario en un momento en el que, precisamente, aumenta el nivel de gastos por la llegada del hijo, hace poco atractivas algunas de las medidas previstas, fundamentalmente las reducciones de jornada y las excedencias ${ }^{13}$. Por otro lado, la inexistencia de permisos dirigidos únicamente a los padres hace que el disfrute acabe recayendo, de forma mayoritaria, en la madre. La situación en la empresa de la mujer trabajadora tampoco facilita la combinación de familia y trabajo. El 29\% de las mujeres trabajadoras de entre

11. Los últimos datos, en las estadísticas que presenta regularmente el Instituto de la Mujer y que pueden consultarse en http://www.mtas.es/mujer/mujeres/cifras/index.htm

12. Pueden consultarse las Estadísticas e Informes que publica el Instituto Nacional de Seguridad Social en http://www.seg-social.es

13. RABANAL CARBAjo, Pedro, escribe que «No se trata de trabajar menos si ello conlleva reducción salarial, pues tal circunstancia conduce necesariamente a variar el estatus socioeconómico familiar. En efecto (...) una norma que permite trabajar menos, pero que conlleva cobrar menos, modifica la situación socioeconómica y por tanto no atiende al fin propuesto, sino que lo altera, pues altera los presupuestos sobre los que se conforma» (Vid. «Familia y trabajo...», op. cit., pág. 56). Lo expresa muy nítidamente De LA VILLA GIL, Luis Enrique: "Usted lo que puede hacer es cambiar su jornada completa por una jornada a tiempo parcial, en el bien entendido que yo le voy a pagar sólo la jornada a tiempo parcial, no la jornada a tiempo completo que usted realizaba antes. Evidentemente, usted tendrá más tiempo para la familia, pero se lo paga usted. (...) Evidentemente, ahí es el propio trabajador el que se está pagando ese esfuerzo de conciliación entre vida laboral y familiar» (Vid. "El marco normativo...", op. cit., p. 73). 
30 y 39 años -franja de edad en la que se vienen teniendo los hijos ${ }^{14}$ - están ligadas a la empresa con contratos temporales ${ }^{15}$, lo que supone, sin duda, un freno a la hora de hacer valer sus derechos ante el empresario. Las que están contratadas indefinidamente se arriesgan a la segura frenada de su carrera profesional ${ }^{16}$, frente a la promoción sin obstáculos del trabajador varón o de la trabajadora sin responsabilidades familiares ${ }^{17}$.

El contexto sociolaboral español tampoco juega a favor de la conciliación entre trabajo y familia. La jornada partida está implantada en muchos sectores de producción, lo que significa un ritmo de trabajo de lunes a viernes, con entre ocho y nueve horas de trabajo diario y una larga pausa para comer, horario totalmente incompatible con las necesidades familiares ${ }^{18}$, máxime si se tiene en cuenta la escasez de centros de atención y cuidado de niños y adultos dependientes. Por otro lado, parece que en España existe todavía la cultura -desterrada ya en países europeos y en EEUU- de la prolongación voluntaria de la jornada, con la conciencia de que salir del trabajo a la hora que correspondería es sinónimo de falta de motivación y, por el contrario, trabajar más horas de las pactadas lo es de dedicación a la empresa, lo que parece premiarse con ascensos y rápida promoción en determinados sectores ${ }^{19}$. Sin embargo, somos el país europeo con menor productividad.

14. Pueden consultarse los datos que presenta GómEz López-EGEA, Sandalio, en su ponencia «La incorporación de la mujer al mercado laboral: implicaciones personales, familiares y profesionales y medidas estructurales de conciliación", en AAVV: La conciliación entre el trabajo y la familia..., op. cit., pp. 110 y 111.

15. Consúltense las estadísticas al respecto disponibles en la web del Instituto de la Mujer (http:// www.mtas.es/mujer/mujeres/cifras/index.htm).

16. Polaino Lorente, Aquilino ofrece el dato de que el $47 \%$ de las mujeres españolas que trabajan consideran que ser madre afecta de forma negativa a su carrera profesional ("La conciliación trabajo/familia y sus implicaciones en la sociedad civil: transformaciones sociales y tendencias de futuro", en AAVV: La conciliación entre el trabajo y la familia.., op. cit., p. 87).

17. Escribe RABANAL CARBAJO, Pedro («Familia y trabajo...», cit., p. 60) que «la existencia de responsabilidades familiares constituye un elemento perjudicial para todo aquél que las asume, pues aunque en el sexo masculino no exista una ostensible discriminación para la contratación, el hombre que asume responsabilidades familiares deja de dedicar a la empresa el 100 por 100 de su tiempo de actividad, con la consecuencia ineluctable de su postergación en el derecho de promoción profesional».

18. Para García ECHeVArRía, Santiago, el del tiempo de trabajo «...es uno de los grandes temas de la economía empresarial actual. En lo que aquí nos concierne, aparte de ser uno de los problemas clave de la configuración de nuestra Sociedad, empuja a la empresa a buscar nuevas formas instrumentales de la organización que sean altamente innovadoras para que puedan devolver a las personas, como tales, una mayor autonomía en la disposición del tiempo.» (Vid. "Exigencias corporativas y estratégicas de la empresa en la conciliación de la vida laboral y familiar», en AAVV: La conciliación entre el trabajo y la familia.... op. cit., p. 50).

19. Vid. CHInChILla Albiol, Nuria, que, como contra-ejemplo, describe el siguiente: «Hace un año SONY quería promocionar a un buenísimo MBA del IESE. El tipo estaba haciéndolo genial, pero se marchaba cada día a las nueve y media de la noche a casa. Entonces le dijeron: "Te queremos promocionar, pero si tú no cambias de actitud y no te marchas antes de las ocho, no podemos promocionarte, porque el mensaje que vamos a dar es que aquí sólo se promociona a la gente que no tiene tiempo para nada más; y eso no nos interesa, así que, por favor, hazlo bien». Le 
Que éste sea el contexto en el que se desarrolla, mayoritariamente, el trabajo por cuenta ajena en nuestro país produce, de forma inevitable, una serie de consecuencias. En primer lugar, para la propia familia. A los trabajadores madres y padres, el desfase entre el horario laboral y el demandado por las necesidades familiares les ocasiona problemas en el trabajo y en el seno familiar, derivados de la falta de tiempo y de la mala calidad del tiempo destinado a atender a la familia, lo que genera estrés, mala calidad de vida y problemas conyugales. Consecuencias también para los hijos, pues se pone de manifiesto cada vez más la ausencia de modelos familiares que reviertan positivamente en la educación de los niños ${ }^{20}$. Y para las empresas, aunque no acaben de interiorizarlo como costo de producción ${ }^{21}$, ya que el estrés que supone para los trabajadores la precaria atención dispensada a la familia, junto con los eventuales retrasos y ausencias derivados del cuidado de los hijos ${ }^{22}$, supone indudablemente un menor rendimiento de los trabajadores ${ }^{23}$. El conjunto de la sociedad también padece las consecuencias derivadas de la dificultad de conciliar empleo y familia. Tenemos uno de los índices más bajos de natalidad mundial, y uno de los más elevados de esperanza de vida ${ }^{24}$ : el envejecimiento de la población activa y su

dejaron tres meses, y cuando hubo cambiado de hábito, le promocionaron» ("Empresa, familia y sociedad, un triángulo en constante evolución", en AAVV: La conciliación entre el trabajo y la familia..., op. cit., p. 129).

20. «Ocho de cada diez mujeres españolas que trabajan están muy contentas con su trabajo, pero la mitad de ellas sostiene que eso influye negativamente en la educación de sus hijos y en las personas que tienen a su cargo.» (Vid. POLAINO LORENTE, Aquilino: Op.cit., p. 87). Datos porcentuales ofrece GÓmeZ LóPEZ-EGEA, Sandalio (Op.cit., p. 115): el 15\% de las mujeres trabajadoras españolas considera que comparte muy poco tiempo con sus hijos; el $46 \%$ cree que es poco tiempo; entiende que comparte un tiempo suficiente el $37 \%$; frente a un $2 \%$ que NS/NC.

21. Más optimista es Polaino Lorente, Aquilino, cuando escribe que «En el ámbito de las empresas se ha despertado una nueva sensibilidad sobre estos problemas. El 90\% de los directores de recursos humanos está de acuerdo en que mejorar la conciliación entre familia y trabajo mejora el rendimiento de los empleados» (Op.cit., p. 85).

22. Los retrasos de la mujer, por motivos familiares, en la hora de entrar al trabajo son del $27 \%$ y en los hombres del $22 \%$. Las salidas anticipadas, por motivos familiares, llegan en las mujeres al $33 \%$ y en los hombres al 22\%, según expone POLAINO LORENTE, Aquilino: Op.cit., p. 87.

23. "Aquellas empresas, y hay mucho ejemplos, que tengan éxito en la innovación del manejo del factor tiempo, vía organización y desarrollo de los recursos humanos, en la integración de los potenciales de mujeres y hombres en el contexto de su actividad, tendrán, sin duda, grandes ventajas competitivas y contribuirán a su propia existencia empresarial»: GARCÍA ECHEVARRÍA, Sandalio: Op.cit., p. 50. El mismo autor escribe que los trabajos en redes, frente a las estructuras jerárquicas y matriciales, «son las únicas formas organizativas que generan los espacios flexibles y los tiempos abiertos para que pueda diseñarse la realización de aportaciones, resultados dentro de equipos de altas prestaciones, que se responsabilizan, tanto por la vía de la organización de proyectos como de procesos. Ésta es, sin duda, la clave del diseño organizativo inmediato que puede acometer la ruptura organizativa tradicional» (ibídem, p. 63).

24. «El incremento vegetativo que experimentó nuestro país hasta hace tres años era deplorable. Se entiende por crecimiento vegetativo el resultado que se obtiene de restar a la suma total de defunciones el número de los nacidos cada año. En el año 1998 el crecimiento vegetativo de nuestro país fue de 4.005; en 1999 de 7.300; y en el año 2000 se elevó a 36.300. Sin embargo, de todos los nacidos, sólo el 3'8\% procedían de padre y madre españolas». Por ello sigue escri- 
escasez son problemas que nos afectan a todos, por su incidencia en el sistema de Seguridad Social ${ }^{25}$.

\section{CONCLUSIONES Y PROPUESTAS}

De lo dicho hasta ahora queda constancia de las siguientes realidades:

1) En las últimas décadas se ha producido una progresiva y pacífica incorporación de la mujer al mundo laboral.

2) Esta incorporación, sin embargo, no ha ido acompañada de una simultánea incorporación del hombre a las tareas domésticas ni de una reacción firme del Estado que facilite de hecho tal incorporación.

3) Las medidas jurídicas que se arbitran en el ámbito de la conciliación trabajo/ familia no son eficaces, ya que, por una parte, restan poder adquisitivo en un momento en el que aumentan los gastos; $y$, por otra parte, porque la empresa se encuentra, con carácter mayoritario, anclada en viejas estructuras y sistemas productivos y de distribución del tiempo de trabajo.

Ante la situación descrita, se hace patente la necesidad de un cambio, tanto legislativo como socio-cultural, que deberá observarse en la totalidad de los agentes implicados, esto es, en primer lugar el legislador, pero junto a él también los agentes sociales (asociaciones de empresarios y sindicatos de trabajadores), la empresa como organización productiva, los varones, las propias mujeres y la sociedad en su conjunto.

Por lo que se refiere al legislador, debe mostrarse más ambicioso en sus objetivos, no limitándose a crear medidas estancas, sino abordando el problema de manera integral, tal y como se ha hecho con la violencia de género. En este sentido debe hacerse especial mención a las normas comunitarias. Así, la Directiva 2002/73/CE contiene el mandato a los Estados Miembros de aplicar el principio de transversalidad en el momento de elaborar las leyes y aplicar las políticas y las actividades de cualquier índole que puedan tener trascendencia sobre la conciliación de la vida laboral y familiar. También constituye responsabilidad del Estado (entendido en sentido amplio, incluyendo a las Comunidades Autónomas que hayan asumido competencias en la materia) la creación de centros de atención y cuidado de mayores y, especialmente, de niños menores de 3 años, aspecto en el que nos encontramos a la cola de Europa y que los textos europeos consideran como instrumento fundamental para la incorporación en condiciones de igualdad de la mujer al trabajo.

Junto a las anteriores, otras medidas que fomentarían la igualdad y, con ello, la conciliación de la vida laboral y familiar, consistirían en premiar o ayudar a través de incentivos fiscales o bonificaciones en la cotización a la Seguridad

biendo POLAINO LORENTE que en el año 2003 se produjo también un incremento significativo del 1'5\% de la población (41.110.000 de españoles), y que ello se explica desde la misma óptica: por la llegada de los inmigrantes y su tasa de natalidad (Op.cit., pp. 94-95).

25. Vid. Rabanal Carbajo, Pedro: "Familia y trabajo...», Op.cit., p. 58. Igualmente, en relación a la crisis del estado del bienestar, centrando el análisis en el contexto de la familia como elemento integrador de aquél, Vid. GARCÍA ECHEVARRÍA, Sandalio: Op.cit., pp. 37-41. 
Social a las empresas que demuestren ser familiarmente responsables, categoría que merecería un reconocimiento público por parte de las autoridades; incentivar la gestión de recursos humanos en las empresas, tanto a través de servicios internos como externos subvencionados; $y$, de manera complementaria a las medidas anteriores, sancionar de forma suficiente los incumplimientos por parte de las empresas en esta materia.

Por otra parte, la Directiva anteriormente mencionada exige también que los Estados Miembros fomenten la participación de los interlocutores sociales en la elaboración y aplicación de medidas al respecto. Este segundo mandato hace obligado preguntarse cómo afronta actualmente la negociación colectiva esta materia.

Pues bien, por lo que se refiere al tratamiento que recibe la materia de conciliación trabajo/familia en los convenios colectivos, la Dirección General de la Mujer de la Comunidad de Madrid ha publicado un riguroso estudio de 100 convenios colectivos, del que se extraen las siguientes conclusiones ${ }^{26}:$ a) los convenios colectivos aplicables institucionalizan el trato desigual, asignando las medidas conciliadoras sólo a las mujeres; b) el lenguaje empleado en las cláusulas relativas a la conciliación confirma esta institucionalización. Es muy frecuente el empleo de términos como «maternidad» y expresiones como «mujer trabajadora» frente a las de «conciliación de la vida familiar y laboral» o "maternidad /paternidad ${ }^{27}$; c) se pone de manifiesto en determinados complementos saláriales una minusvaloración del trabajo realizado por la mujer, por el sólo hecho de serlo; d) prácticamente son inexistentes los complementos que tienden a lograr el $100 \%$ del salario en las situaciones de baja por riesgo durante el embarazo.

Los resultados de este análisis ponen de manifiesto una clara falta de asunción por parte de empresarios y sindicatos de su responsabilidad en esta materia, que queda prácticamente limitada a la demanda y exigencia de medidas a

26. Guía de Criterios y Cláusulas para la Negociación de los convenios, en materia de Conciliación de la Vida Familiar y Profesional, Madrid, Comunidad de Madrid, 2003.

27. Como indica Menéndez Sebastián «...es preciso que los interlocutores (...) formulen en general los derechos sin distinción por razón de sexo, evitando que la trabajadora reciba un trato más beneficioso o perjudicial cimentado únicamente en su condición femenina...» La autora propone, en este sentido, que el derecho a la ausencia por lactancia (art. 37.4 ET) se reconozca en los convenios como exclusivo de la madre sólo si la alimentación se va a producir de forma natural. De esta manera, el empresario no tiene porqué presumir que será la mujer quien disfrute de la reducción salvo en el supuesto señalado (la autora reseña un listado de convenios que así lo recogen, si bien reconociendo su carácter excepcional). Otro ejemplo de la infrautilización de la negociación colectiva como vía de igualdad puede apreciarse al observar que los convenios colectivos no corrigen la tendencia a presuponer que el derecho a permisos para preparación al parto son titularidad de la mujer, lo que resulta disuasorio para la contratación de éstas. (MENÉNDEZ SEBASTIÁN, Paz: "Principio de igualdad y configuración convencional de las reducciones de jornada y los permisos específicos para la conciliación de la vida familiar y laboral», en AAVV, Nueva sociedad $y$ derecho del trabajo, op.cit., pp. 632 y ss). 
la propia administración ${ }^{28}$. No obstante, se observan, paralelamente, cláusulas en convenios colectivos que sí pueden ser objeto de una valoración positiva. Así ocurre con aquellas que, en igualdad de condiciones de idoneidad, declaran la preferencia por las personas del género menos representado en el grupo profesional (vid., al respecto, Convenio Colectivo de la Industria Química y Convenio de las industrias transformadoras de plásticos de la COM. Aut. De Madrid).

Por otro lado, la empresa, no ya a través de sus representantes colectivos, sino como entidad individualmente considerada, es el lugar en el que las medidas previstas legal y convencionalmente deben ser aplicadas. Una aplicación eficaz de las mismas requiere una adecuada organización del trabajo. Al respecto, resulta extraordinariamente significativo el hecho de que nuestro país sea el menor índice de productividad de Europa. Cada empresario ha de asumir que un trabajador satisfecho con su vida personal porque dispone de un horario de trabajo que le permite atender cualitativa y cuantitativamente a su familia es un trabajador que incrementa su productividad, reduciendo errores y padeciendo un menor número de accidentes/incidentes; en definitiva, es más rentable. Un cambio en la política legislativa que no venga acompañado de otro en la cultura empresarial estará abocado al fracaso, ya que ningún trabajador hará uso de las medidas que el Estado pone a su disposición por temor a represalias que afecten a sus posibilidades de promoción o incluso de conservación de su empleo. En consecuencia, debe generalizarse la implantación de planes internos de conciliación de vida familiar y laboral -los llamados Programas de Trabajo/Familia-, a través, fundamentalmente, de la flexibilidad laboral. Los horarios estrictos y el control de horas presenciales deben ser sustituido por horarios flexibles, autocontrol y una evaluación del rendimiento basada en objetivos, no en horas de trabajo ${ }^{29}$. Las empresas también deben empezar por sí mismas a facilitar la vida familiar de sus trabajadores, desde la perspectiva de que, así, rendirán más ${ }^{30}$.

Aunque desde este estudio se insiste en que la conciliación de vida laboral y familiar no es un problema específico de las mujeres, lo cierto es que, de hecho, la incorporación de mujeres a órganos directivos de las empresas constituye importante baza a tener en cuenta, ya que una mujer directiva (o empresaria) tiende, hoy por hoy, a mostrar una mayor sensibilidad respecto de estos problemas.

28. Tras constatar esta realidad, De La Villa Gil propone la creación de fondos de cofinanciación de acciones a favor de la conciliación familiar-laboral sufragada por empresas y trabajadores por medio de la negociación colectiva, sin que ello desmerezca el hecho de que el gran coste de la conciliación deba grabar a los poderes públicos (DE LA VILLA GIL, Luis Enrique: «El marco normativo...", op.cit., pp. 79 y ss).

29. En este sentido, IBM España constituye un ejemplo a seguir de empresa con horarios flexibles y gestión por objetivos.

30. Airtel, por ejemplo, fue de las primeras empresas que ofrecieron en España la opción de una baja por maternidad más larga de la que la ley ofrece; también ofrece a los padres cinco días -en vez de los dos legales- para atender a un hijo recién nacido. 
No obstante, ni las medidas legislativas ni el cambio en la organización del trabajo en las empresas serán efectivos si no van acompañados de un cambio profundo en la mentalidad de los varones españoles, que deben incrementar su grado de implicación tanto en el cuidado de los hijos y/o familiares como en el cumplimiento de las tareas domésticas. Este cambio concreto en la mentalidad de los hombres presenta importantes dificultades para su promoción desde las medidas legislativas (aunque sí sería posible, por ejemplo, establecer como medida permisos de los que pudiera disfrutar sólo el padre) y constituye sin duda un pilar fundamental para la efectiva conciliación de la vida familiar y laboral

Ahora bien, en ocasiones, el obstáculo se encuentra en la propia concepción que determinadas mujeres tienen respecto del rol que deben asumir en la sociedad, en el trabajo y en su familia. En este sentido, no sólo la mujer de ámbito rural, escasamente formada, sino también -aunque parezca sorprendente- la mujer urbana, con formación académica y profesional, acepta e institucionaliza en su propia familia su doble rol de trabajadora y madre, asumiendo casi en exclusiva las tareas domésticas. El peligro no es sólo actual, sino también de futuro, dado que ello supone educar a los hijos de hoy-adultos de mañana- en la perpetuación de la división sexual del trabajo. Por este motivo son tan importantes los movimientos asociativos dirigidos a exponer, analizar y cambiar la situación laboral y familiar de la mujer, por la capacidad que los foros sociales tienen para llegar hasta el individuo.

Para finalizar, se debe hacer una llamada a toda la sociedad, en cuanto sociedad consumista. Cada uno de sus miembros tenemos no sólo la responsabilidad sino también el poder de premiar con nuestro consumo a aquellas empresas con certificado de EFR (Empresa Familiarmente Responsable) y, simultáneamente, retirar nuestro apoyo a las que no lo son y, especialmente, a las incumplidoras u obstaculizadoras. Esta es una forma de denuncia pública altamente eficaz.

Conseguir, en fin, que la vida familiar y laboral resulten compatibles es -aunque suene a tópico- una tarea de todos, no sólo del legislador. 